\title{
Synthesis and Characterization of Plasmonic Resonant Guided Wave Networks
}

\author{
Stanley P. Burgos, ${ }^{\dagger, \dagger}$ Ho W. Lee, ${ }^{\dagger, \dagger}$ Eyal Feigenbaum, ${ }^{\dagger}$ Ryan M. Briggs, $^{\dagger}$ and Harry A. Atwater ${ }^{*}, \dagger$ \\ ${ }^{\dagger}$ Thomas J. Watson Laboratories of Applied Physics and ${ }^{\ddagger}$ Kavli Nanoscience Institute, California Institute of Technology, $1200 \mathrm{E}$ \\ California Blvd, Pasadena, California 91125, United States
}

\section{Supporting Information}

ABSTRACT: Composed of optical waveguides and powersplitting waveguide junctions in a network layout, resonant guided wave networks (RGWNs) split an incident wave into partial waves that resonantly interact within the network. Resonant guided wave networks have been proposed as nanoscale distributed optical networks (Feigenbaum and Atwater, Phys. Rev. Lett. 2010, 104, 147402) that can function as resonators and color routers (Feigenbaum et al. Opt. Express 2010, 18, 25584-25595). Here we experimentally characterize a plasmonic resonant guided wave network by demonstrating that a $90^{\circ}$ waveguide junction of two v-groove channel plasmon polariton (CPP) waveguides operates as a compact powersplitting element. Combining these plasmonic power splitters with CPP waveguides in a network layout, we characterize a prototype plasmonic nanocircuit composed of four v-groove waveguides in an evenly spaced $2 \times 2$ configuration, which functions as a simple, compact optical logic device at telecommunication wavelengths, routing different wavelengths to separate transmission ports due to the resulting network resonances. The resonant guided wave network exhibits the full permutation of Boolean on/off values at two output ports and can be extended to an eight-port configuration, unlike other photonic crystal and plasmonic add/drop filters, in which only two on/off states are accessible.

KEYWORDS: Plasmonics, nanocircuits, resonant structures, power splitters, silicon photonics, logic devices
$\mathrm{I}_{\mathrm{n}}^{\mathrm{t}}$ $\mathrm{t}$ has recently been proposed that resonant guided wave networks (RGWNs) can engineer dispersion through wave resonances in a waveguide network consisting of waveguides coupled at their intersections. ${ }^{1-3}$ Two basic elements form the network: waveguides and power-splitting waveguide junctions, which redistribute partial waves within the network. The RGWN concept can be implemented in various network configurations, e.g., using optical fibers or $\mathrm{Si}$ rib waveguides in planar $\mathrm{Si}$ photonic structures, ${ }^{4,5}$ or metal/dielectric/metal (MDM) slab, or coaxial plasmonic waveguides. ${ }^{6-12}$ However, excitation of distributed resonances in the network by interaction of partial waves relies on the realization of a power-splitting element, which is nontrivial to achieve in dielectric photonic structures ${ }^{13}$ since an intersecting junction between two photonic waveguides will not lead to a four-way power splitting, due to the $k$-vector mismatch between the incoming wave and the desired outgoing waves. ${ }^{1,2,13,14}$

It has been proposed that the crossing of two plasmonic MDM slab waveguides can function as a power splitting element for a plasmonic resonant guided wave network. In this configuration, a highly confined waveguide mode with a welldefined high $k$-vector enters the junction from one of its ports. In the waveguide junction, the mode expands into an outgoing wave with isotropic in-plane distribution of $k$-vectors due to volumetric expansion of the mode as it enters the junction, and the outgoing wave couples into all four ports of the waveguide crossing, leading to a power-splitting scattering event. ${ }^{1-3,15}$ For the case of a four-way $90^{\circ}$ waveguide intersection, it has been theoretically shown that the amplitude and phase of the waves that are coupled back into the waveguide arms of the junction can be tuned by varying the size of the dielectric gap, and that certain designs can facilitate equal power splitting into all four output ports. $^{1-3,15}$

MDM plasmonic slab waveguides of infinite extent can be used to conceptually motivate the resonant wave interactions, but reduction to experimental practice requires the RGWN to be rendered as a network of waveguides with a finite cross section, such as a slot, or v-groove, MDM waveguide for experimental implementation. ${ }^{1}$ The confined v-groove slot waveguide mode (channel plasmon polariton, CPP) is similar to that of the infinite MDM slab waveguide both in terms of polarization and field confinement, ${ }^{16-23}$ which are required properties for the realization of power splitting. However, the vgroove slot waveguide configuration also supports a surface plasmonic mode (SPP) that is confined at its corners, ${ }^{17,23}$ so care must be taken to properly excite the desired CPP waveguide mode instead of the SPP mode in a v-groove slot waveguide. To address this concern, we excited the v-groove

Received: February 24, 2014

Revised: May 15, 2014

Published: May 20, 2014 


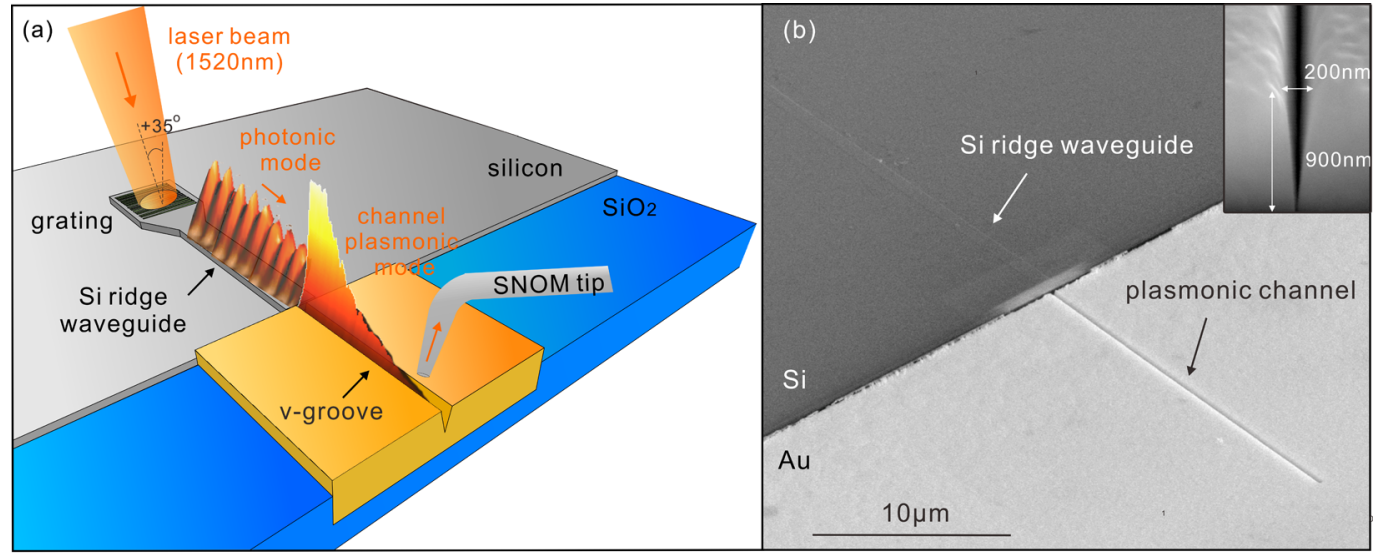

(c) Channel plasmon polariton (CPP)

(d) Surface plasmon polariton (SPP)

(e) Silicon photonic mode
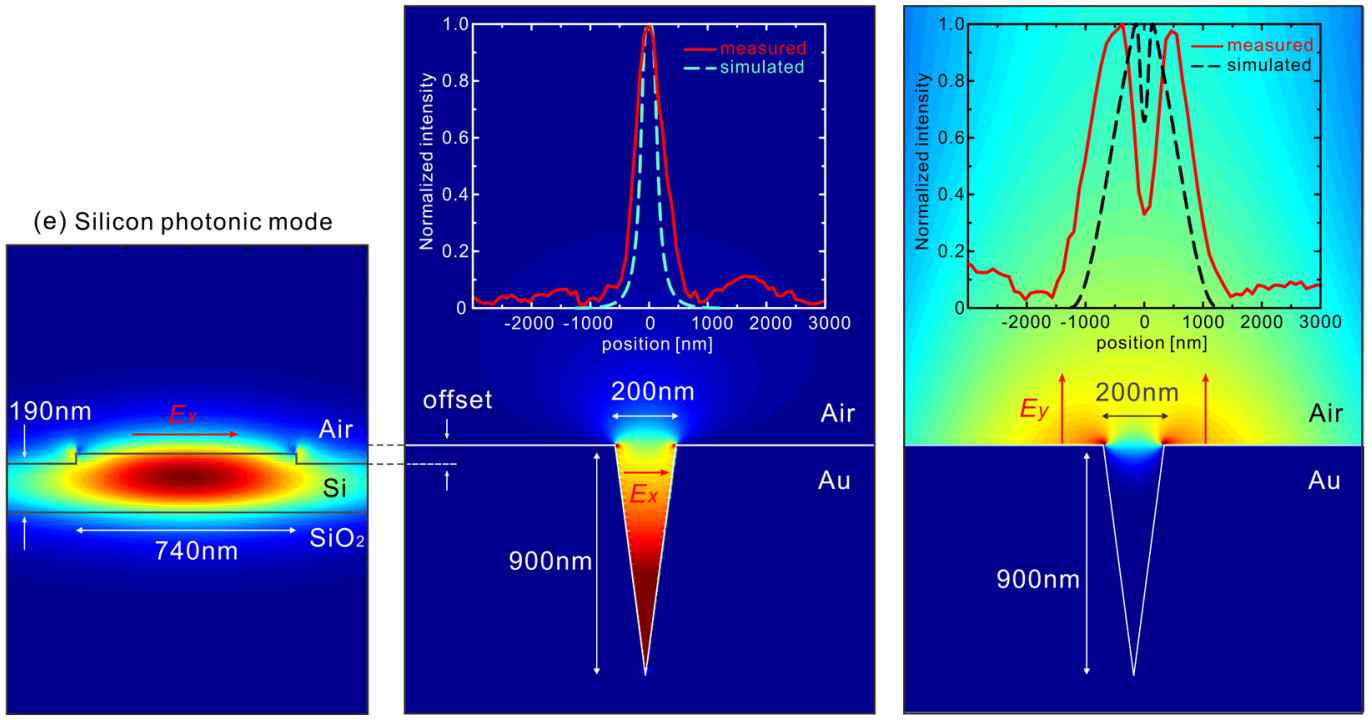

Figure 1. Selective excitation and near-field imaging of Si-coupled v-groove plasmonic waveguide mode. (a) Schematic of Si-photonic/v-groove plasmonic hybrid device and illumination condition used. (b) Scanning electron micrograph (SEM) of a Si-ridge/v-groove hybrid device fabricated with electron beam lithography and focused ion beam milling. Inset: SEM cross-section of the $200 \mathrm{~nm} \times 900 \mathrm{~nm}$ v-grove waveguide. (c,d) Calculated electric field distributions for CPP $\left(E_{x}\right)$ and SPP $\left(E_{y}\right)$ modes supported inside and near the surface of the v-groove. Insets to (c,d): Corresponding measured and calculated field intensity profiles for CPP and SPP modes at a height of $75 \mathrm{~nm}$ above the surface of the v-groove. Dashed line scans correspond to calculations, and solid line scans correspond to near-field optical measurements. (e) Electric field distribution of TE mode in the Siridge waveguide used to excite the CPP mode of the v-groove.

slot waveguide from a silicon-on-insulator waveguide with a butt-coupled junction in which the proper silicon-on-insulator waveguide mode is launched so as to selectively couple into the desired CPP mode of the plasmonic slot waveguide (Figure 1).

We experimentally demonstrate that the CPP mode of the $\mathrm{v}$ groove waveguide can be selectively excited by mode polarization control in the silicon-on-insulator waveguide, which suppresses excitation of the SPP mode. Once the incident wave is coupled into the proper channel plasmon polariton mode of the plasmonic v-groove waveguide, we experimentally demonstrate for the first time that a $90^{\circ}$ fourway waveguide junction operates as an ultracompact powersplitting element. To characterize network power splitting, we fabricated a plasmonic nanocircuit composed of four v-groove waveguides in an evenly spaced $2 \times 2$ configuration. This structure can function as a compact optical logic device at telecommunication wavelengths, routing different wavelengths via different on/off combinations to separate transmission ports. The prototype logic device reported here exhibits eightport operation, unlike other photonic crystal and plasmonic add/drop filters, in which only two on/off states are accessible. $^{24,25}$ This work experimentally illustrates how ultracompact plasmonic components can be selectively excited with Si photonics, creating the platform for next-generation integrated plasmonic circuits.

Plasmonic Modes of the v-Groove Structure. The vgroove channel plasmon waveguide configuration ${ }^{16-23}$ supports two fundamental plasmonic modes, the confined slot plasmon polariton (CPP) (Figure 1c), residing in between the v-groove sidewalls, and the surface plasmon mode (SPP), which is a delocalized surface wave weakly bound at the top of the vgroove geometry by its corners (Figure 1d). As evident from Figure $1 c, d$, the CPP mode is characterized by an electric field perpendicular to the v-groove sidewalls (similar to the TM metal/dielectric/metal plasmonic mode), while the SPP mode is characterized by an electric field perpendicular to the top of the v-groove surface (similar to that of a regular SPP mode). ${ }^{26}$ Thus, these two modes differ not only in modal volume and confinement but are also characterized by orthogonal polar- 
(a)
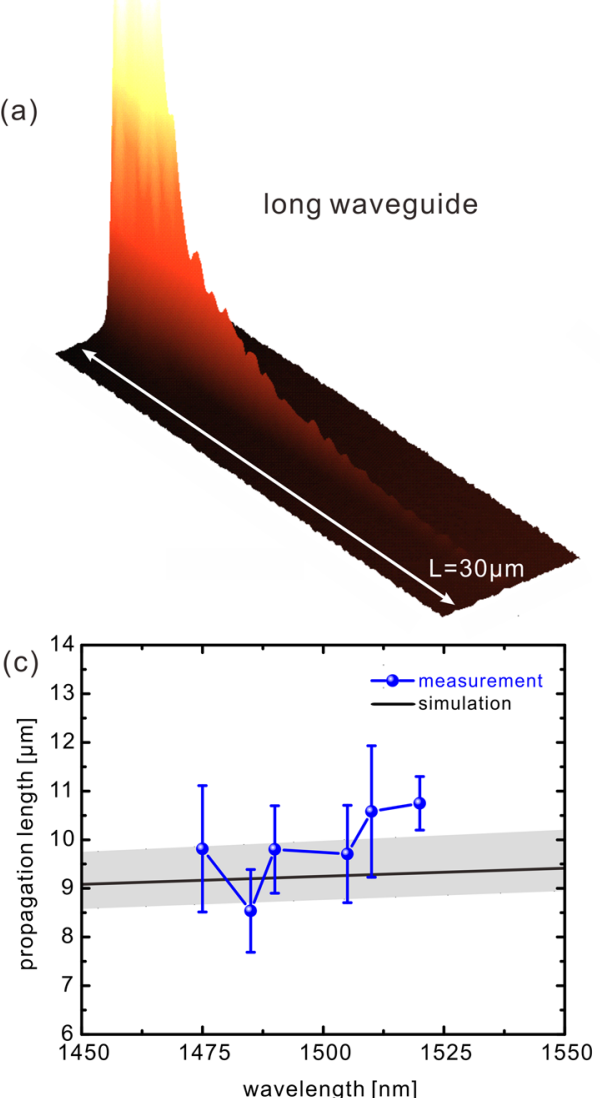

(b)

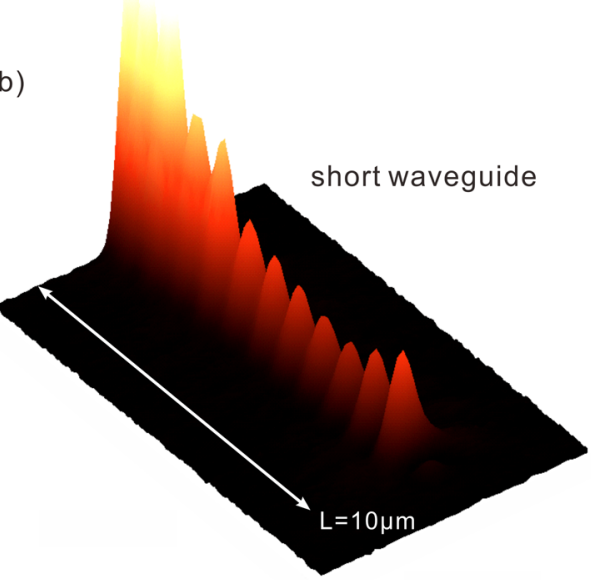

(d)

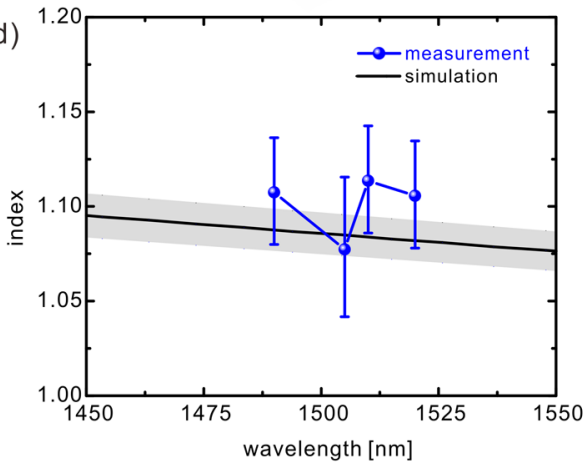

Figure 2. NSOM images of CPP v-groove waveguide mode. Experimental near-field scanning optical microscope image taken at $\lambda_{0}=1520 \mathrm{~nm}$ of $(\mathrm{a})$ $30 \mu \mathrm{m}$ and (b) $10-\mu \mathrm{m}$-long v-groove waveguides. (c) Propagation length of v-groove waveguide mode obtained from full-field electromagnetic simulations (black line) (shaded areas indicate the results with $\pm 10 \mathrm{~nm}$ variation on the v-groove width) and by fitting the decaying near field intensities of long v-groove waveguides (blue dotted data). (d) Effective index of v-groove waveguide mode derived from eigenmode solver calculations (black line) and the standing wave pattern observed in near field intensity measurements of short v-groove waveguides (blue points).

ization, allowing for polarization-based selectivity between the two modes.

The mode confinement also manifests itself in the propagation length and the effective index of the two modes, with the CPP mode having a propagation length of $L \sim 10 \mu \mathrm{m}$ and effective index of $n \sim 1.1$ at $\lambda_{0}=1520 \mathrm{~nm}$, an order of magnitude shorter than that of the SPP with $L>100 \mu \mathrm{m}$ and an effective index of $n \sim 1.0$. This propagation length difference means that if we were to simply excite the v-groove plasmonic waveguide from free space in an end-coupled configuration and measure the intensity down the v-groove waveguide past the scattering-dominated area or at a waveguide output, we would only be able detect the SPP mode owing to its longer propagation length. However, the SPP mode is weakly confined and therefore does not exhibit network resonances since isotropic power splitting does not occur in the waveguide junctions for this mode. Thus, an alternative guided wave coupling scheme is required to selectively couple into the CPP mode, motivating our use of $\mathrm{Si}$ photonic waveguide coupler.

Plasmonic Waveguide Excitation from a Si Photonic Waveguide. We fabricated a hybrid Si-photonic/v-groove plasmonic chip consisting of $300 \mu \mathrm{m}$ long Si-ridge waveguides end-coupled to v-groove channel plasmon polariton waveguides of variable length and layout (see Figure 1a,b) (see methods for fabrication details). The v-groove channel waveguides were fabricated using multipass focused ion beam (FIB) milling. The typical dimensions of the v-groove waveguide were $200 \mathrm{~nm}$ wide and $900 \mathrm{~nm}$ deep, which were measured by taking FIB cross-section images of the structure (see inset of Figure $1 \mathrm{~b}$ ). Telecommunication wavelength light from a tunable diode laser was coupled into the Si-ridge waveguide through a grating coupler fabricated far away from the Si-ridge/v-groove interface, thus separating the on-chip coupling from the excitation of the v-groove mode. The grating coupler was designed to selectively couple light into either the TE (E-field parallel to the substrate, see Figure 1e) or TM (E-field perpendicular to the substrate) modes of the Si-ridge waveguide by controlling the angle of excitation on the Siridge grating, due to the different phase velocities of the TE and TM modes. For example, by illuminating with $\lambda_{0}=1520 \mathrm{~nm}$ light at an incident angle of $+35^{\circ}$ (Figure 1a) and adjusting the polarization of the incident light, we were able to access the Siridge TE mode, which matches the dominant E-field distribution of the CPP mode (see Figure 1c), thus allowing us to selectively couple into this mode based on polarization selectivity alone. The optical response of the system was measured using a Nanonics MV2000 near-field scanning optical microscope operating in collection mode. A near field optical probe was scanned over the Si-ridge waveguide and plasmonic resonant waveguide network, collecting the near-field intensity of the evanescent wave at the surface of the v-groove plasmonic waveguide, which was recorded as a function of position with an InGaAs photodiode. From the NSOM measurements of the Si-ridge/v-groove interface, the amount of light coupled from 
(a)

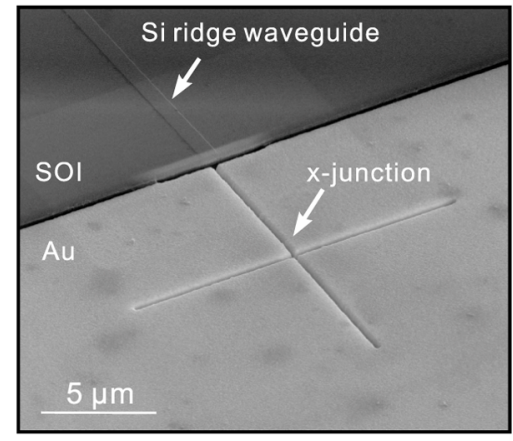

(c) ${ }^{29}$

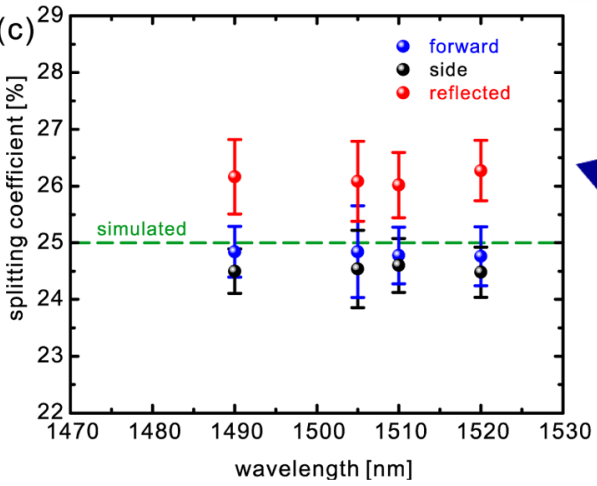

(b)

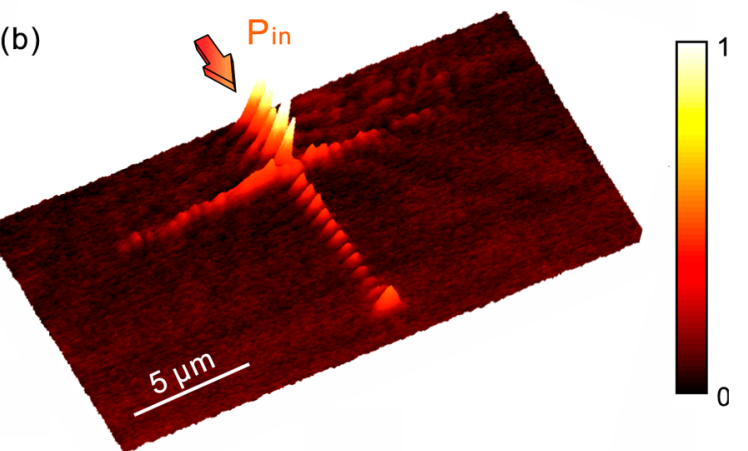

(d)

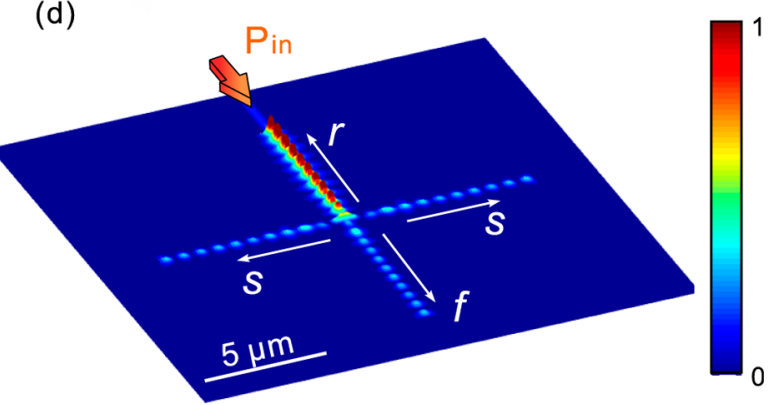

Figure 3. Equal-power-splitting v-groove waveguide $\mathrm{x}$-junction plasmonic structure. (a) Scanning electron micrograph of two v-groove waveguides crossed at $90^{\circ}$, forming a waveguide junction coupled from one of its ports by a Si-ridge waveguide. (b) Near-field scanning optical image taken at $\lambda_{0}$ $=1520 \mathrm{~nm}$ of the v-groove waveguide $\mathrm{x}$-junction shown in (a). (c) Power splitting parameters extracted from fitting the intensity pattern of the near field image shown in (b). (d) Simulated optical response of v-groove waveguide crossing excited through one of its ports with the CPP mode at $\lambda_{0}=$ $1520 \mathrm{~nm}$.

the Si-ridge waveguide into the v-groove CPP waveguide was calculated by measuring the near field intensity distribution at the Si-ridge/v-groove waveguide junction and using full-field electromagnetic simulations (finite-difference time-domain calculations) to relate the intensities to the power in the waveguides. By comparing the intensities before the $\mathrm{Si}$ waveguide/plasmonic waveguide junction (including contributions from the incoming and reflected photonic modes of the Si waveguide) and after the junction (which accounts for the intensity coupled into the CPP waveguide mode), we calculate a coupling efficiency of $\sim 22.2 \%$ (see Methods for the details of calculation). This coupling efficiency, although not ideal, demonstrates the successful integration of conventional silicon photonics with highly confined v-groove plasmonic modes, the first steps toward realizing advanced integrated $\mathrm{Si} /$ plasmonic nanocircuits. It should be noted that the coupling efficiency could be significantly enhanced with more sophisticated engineering, such as multisection tapers and stub-resonant structures $^{27-34}$ (see Supporting Information for more detail on coupling efficiency and calculation).

CPP Waveguide Mode Properties. The key physical parameters of the CPP waveguide can be determined from its modal properties, namely, propagation length, effective index, and modal intensity full-width half-max (FWHM), at the device surface. The CPP propagation length is obtained by measuring the field decay of the light down the $30 \mu \mathrm{m}$ long v-groove length (Figure 2a) starting at the coupling point from the Siridge waveguides. By fitting a decaying exponential to the measured intensity variation along the CPP waveguide, we measure a propagation length of $L \sim 10 \mu \mathrm{m}$ for $\lambda_{0}=1520 \mathrm{~nm}$, consistent with full field electromagnetic simulations for the
CPP mode propagation length. The resulting CPP propagation length as a function of excitation wavelength is plotted in Figure 2c, demonstrating good agreement with the calculated CPP eigenmode of the v-groove structure (black curve). The shaded areas indicate the simulated values with $\pm 10 \mathrm{~nm}$ variation of the v-groove width.

The effective mode index of the structure is obtained from measurements of the standing wave intensities for $10-\mu \mathrm{m}$-long waveguides, a waveguide length comparable to the propagation length (Figure $2 \mathrm{~b}$ ). The resulting longitudinal resonances give rise to standing-wave interference patterns of different periodicity for different excitation wavelengths, and this intensity pattern can be fitted to extract the effective index of the waveguide mode. From this fit, we extract an effective index of $n \sim 1.11$ at $\lambda_{0}=1520 \mathrm{~nm}$, consistent with the index of a bound mode $(n>1)$. The resulting mode effective index as a function of wavelength is plotted in Figure $2 \mathrm{~d}$, demonstrating good agreement with the eigenmode calculation of the CPP mode dispersion. The slight discrepancy may be attributed to the small geometry and dielectric constant difference between the simulated and the experimental structures.

Furthermore, in addition to the differences in propagation lengths, the SPP and CPP modes also differ in their modal cross sections, with the CPP having a single transverse FWHM of only $300 \mathrm{~nm}$, and the SPP having a double peak with a total transverse FWHM of $>1 \mu \mathrm{m}$ (insets of Figure 1c,d). To definitively validate that we have selectively coupled light into the CPP mode of the v-groove channel, we also measured the FWHM of the NSOM intensity profiles obtained at cross sections of the v-groove waveguide when excited with TE polarized light from the $\mathrm{Si}$ waveguide. A typical line scan at 


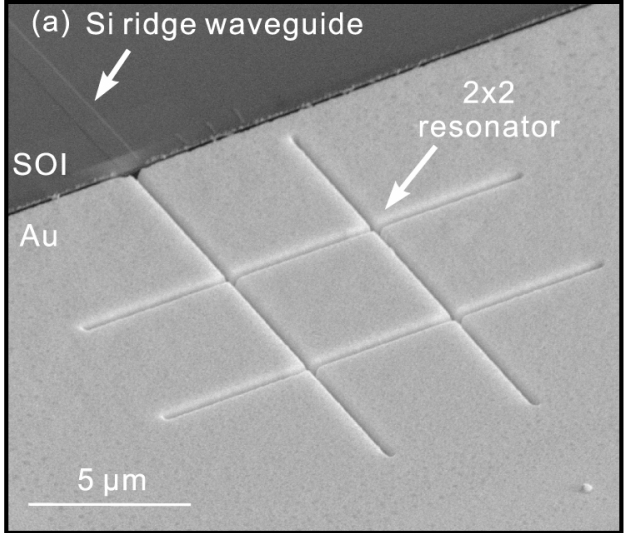

(off/off state)

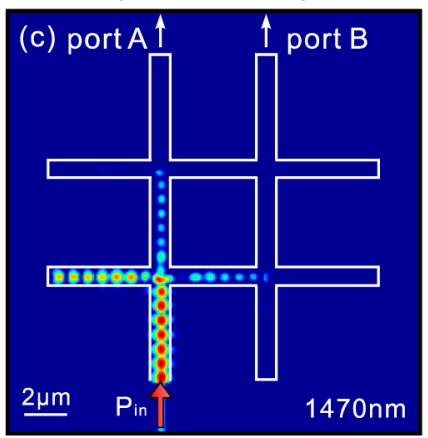

(on/off state)
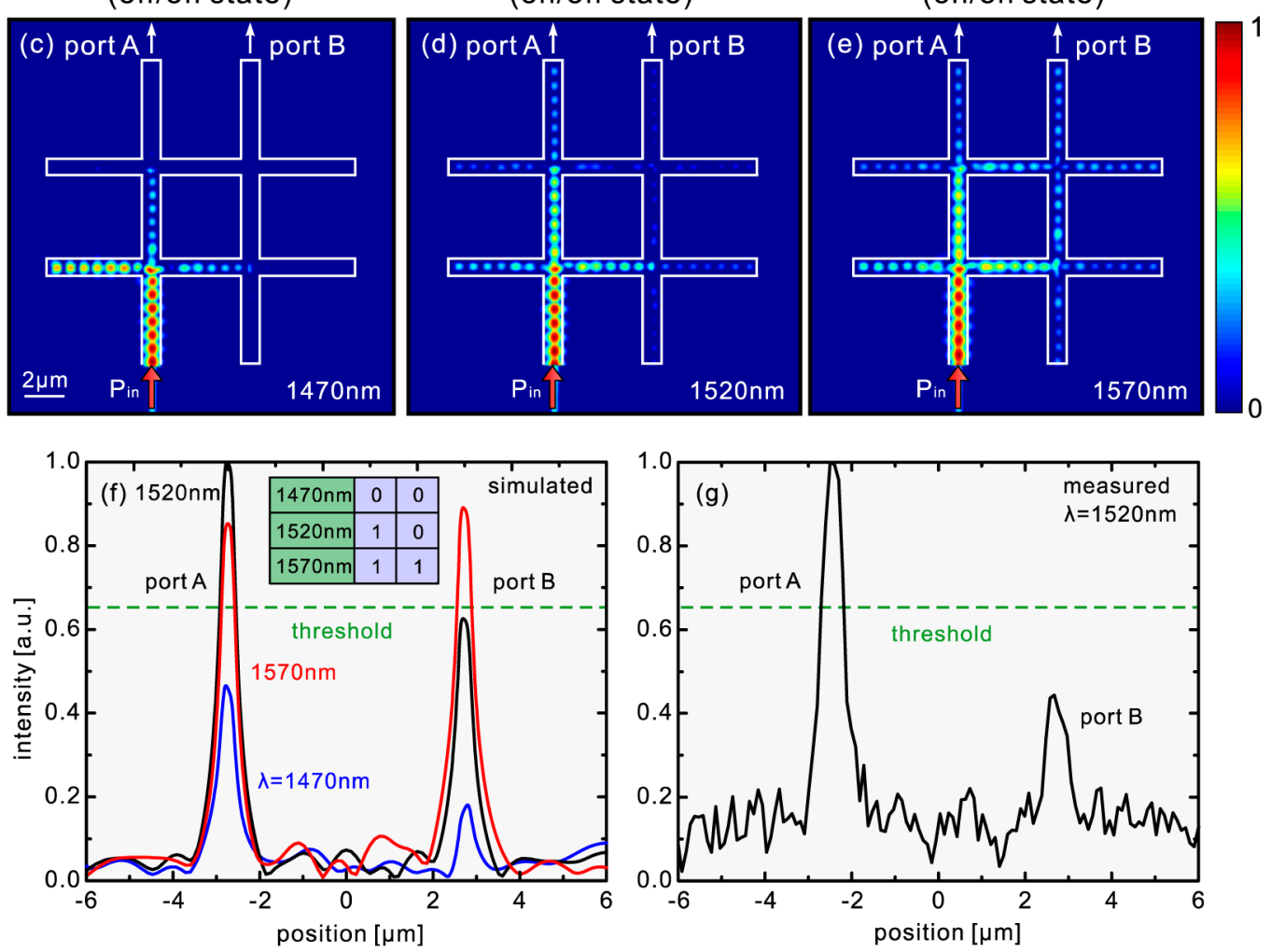

Figure 4. $2 \times 2$ resonant guided wave network logic device. (a) Scanning electron micrograph of fabricated device consisting of four $15 \mu \mathrm{m}$ long waveguides in an evenly spaced $2 \times 2$ configuration, coupled from one of the arms with a Si-ridge waveguide. (b) Measured near field response of device shown in (a), when excited with $\lambda_{0}=1520 \mathrm{~nm}$ TE polarized light from the Si-ridge waveguide. The output ports of interest (A and B) are labeled for reference. $(\mathrm{c}-\mathrm{e})$ Simulated near-field intensity of plasmonic logic device excited with the CPP mode from the bottom left port at $(\mathrm{c}) \lambda_{0}=$ $1470 \mathrm{~nm},(\mathrm{~d}) \lambda_{0}=1520 \mathrm{~nm}$, and (e) $\lambda_{0}=1570 \mathrm{~nm}$. The top ports on interest are labeled along with their on/off state configuration based on the excitation wavelength. (f) Intensity cross sections taken at top output ports of interest at the excitation wavelengths shown in ( $c-e)$. (g) Measured intensity response at the output ports of interest for $\lambda_{0}=1520 \mathrm{~nm}$, showing the on/off Boolean state.

$1520 \mathrm{~nm}$ is shown in Figure 1c (solid red curve), showing a single peak FWHM of $\sim 400 \mathrm{~nm}$, which is only slightly larger than that predicted by full-field electromagnetic simulations. We attribute the difference between the calculated and experimental values of the transverse modal FWHM to the convolution of the evanescent tail of the mode with the resolution of NSOM tip collection aperture. Note that, compared with the silicon dielectric mode which becomes unconfined for widths below $\sim 700 \mathrm{~nm}$, the v-groove CPP mode is a truly subwavelength mode and is thus the only possible mode to achieve equal power splitting and resonant network (see Supporting Information for silicon dielectric waveguide mode).

We can also access the SPP mode of the structure by changing the excitation angle on the Si-ridge grating. By rotating the excitation angle to $-35^{\circ}$ (Figure 1a), we are able to access the TM mode of the Si-ridge waveguide, which matches the E-field profile of the SPP mode of the v-groove waveguide (Figure 1d). By coupling in this manner, and measuring the resulting near field distribution along the v-groove waveguide structure, we obtain the line scan shown in Figure 1d (solid curve), demonstrating that the measured mode is indeed double-peaked with a FWHM $>1 \mu \mathrm{m}$, consistent with the 
simulated field distribution of the SPP mode. Thus, by proper control of the polarization in the Si-ridge structure we can access either the CPP or SPP mode of the v-groove structure. However, only the CPP mode exhibits truly subwavelength transverse mode confinement, important to the design of RGWN structures and other compact plasmonic circuits. ${ }^{1-3}$

Ultracompact Four-Way Power-Splitters. A plasmonic power splitting waveguide junction can be realized by the intersection of two subwavelength mode CPP waveguides. Figure 3a is a scanning electron microscopy image of a power splitting junction, consisting of two $15-\mu \mathrm{m}$-long v-groove waveguides crossed at their centers at a $90^{\circ}$ angle. The waveguide junction is excited from one of its ports at $\lambda_{0}=1520$ $\mathrm{nm}$ with a TE mode of the $\mathrm{Si}$ waveguide, thus coupling into the CPP mode as demonstrated in the single waveguide measurements described previously.

The resulting NSOM image of the waveguide junction is shown in Figure 3b, from which we can see that power is split among the four ports of the junction. We also observe standing wave interference patterns in each of the waveguide arms, with that of the forward and sideways ports (relative to the excitation port) arising from the waveguide-end reflections and that of the excitation port coming from the reflection at the waveguide junction. To extract the amount of power coupled into each port, we fit the resulting intensity patterns in each of the waveguide arms, getting approximately equal power splitting into each port at $\lambda_{0}=1520 \mathrm{~nm}$ (see Figure 3c). We note that, although the waveguide junction scattering coefficients are complex, we are only able to extract their intensities from our experimental measurements since our NSOM setup is not equipped to detect phase. Nevertheless, we determine the dispersion of the splitting coefficient amplitudes by varying the incident wavelength and repeating the amplitude fitting routine described above. The resulting data as a function of wavelength are plotted in Figure $3 c$, demonstrating equal power splitting amplitudes that are fairly insensitive to wavelength over a range of $30 \mathrm{~nm}$. Note that the error bars in Figure $3 c$ result from the uncertainly of the fitting parameters. It is also important to mention that the equalpower splitting only occurs in a truly subwavelength plasmonic splitter. For a purely dielectric waveguide crossing without any additional scattering element (such as a silicon waveguide crossing), most of the energy entering the waveguide junction will propagate in the forward direction without splitting to the side ports (see Supporting Information for details of this calculation for a dielectric waveguide crossing).

To substantiate our findings, we investigate the expected splitting amplitudes using FDTD simulations, which are superimposed as a false color map onto the measured data in Figure $3 c$, yielding good agreement between measurement and theory. For visual comparison, in Figure 3d, we also plot the simulated field intensity profile of a $200 \times 900 \mathrm{~nm}$ v-groove waveguide junction. A CPP mode is launched from one of the waveguide junction arms, and the resulting steady state field is recorded at the surface of the structure. We observe from Figures $3 b, d$ that both near-field spectra are in good agreement of each other. It should be noted that the less confined SPP mode behaves differently and does not split at the waveguide junction (see details in Supporting Information, Figure S3).

$2 \times 2$ Port Plasmonic Logic Device. As a demonstration of one possible plasmonic circuit enabled by the presented subwavelength waveguides and splitters, we fabricated a plasmonic network composed of four $15 \mu \mathrm{m}$ v-groove waveguides in a $2 \times 2$ configuration (see Figure $4 a$ ), which is shown to operate as a compact optical logic device at telecommunication wavelengths, routing different wavelengths with different on/off combinations to different transmission ports.

First, we investigate the $2 \times 2$ waveguide network properties using full-field electromagnetic simulations, where we excite the bottom left port $\left(\mathrm{P}_{\mathrm{in}}\right)$ with a broadband CPP mode and monitor the output at the two top ports (port A and B) of the structure for all of the wavelengths (see Supporting Information for all seven ports). Setting the threshold detected intensity to 0.65 , from the selected near-field distributions in Figures $5 \mathrm{c}-\mathrm{e}$,
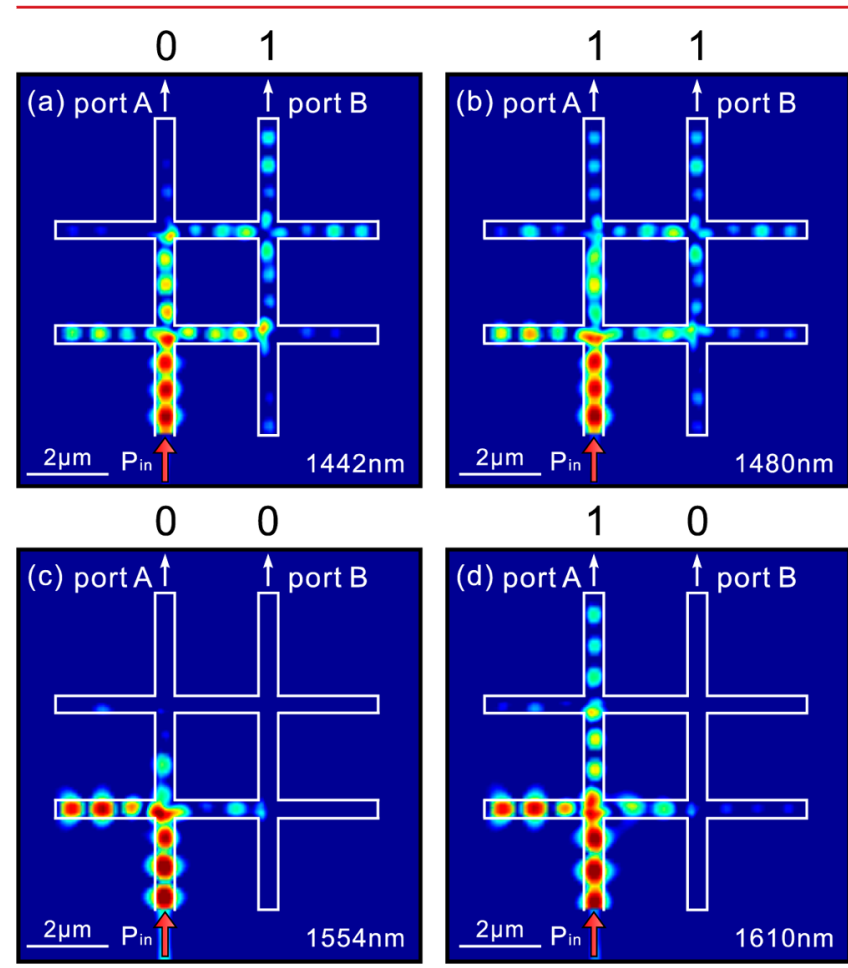

Figure 5. Full Boolean permutations of the $2 \times 2$ network taken at the two output ports. Simulated power distribution of $2 \times 2$ network with smaller dimensions $(7.5 \mu \mathrm{m} \times 7.5 \mu \mathrm{m})$ at the following wavelengths (a) $\lambda_{0}=1442 \mathrm{~nm}$, (b) $\lambda_{0}=1480 \mathrm{~nm}$, (c) $\lambda_{0}=1554 \mathrm{~nm}$, and (d) 1610 $\mathrm{nm}$. The top ports (A and B) are labeled along with their on/off state configuration at each excitation wavelength, demonstrating that a full set of Boolean on/off states can be achieved for Ports A and B.

we observe that $\lambda_{0}=1520 \mathrm{~nm}$ light selectively routs to the top left port (on/off configuration, Figure 4d), $\lambda_{0}=1570 \mathrm{~nm}$ routes to both top left and top right ports (on/on configuration, Figure $4 \mathrm{e}$ ), $\lambda_{0}=1470 \mathrm{~nm}$ routes to neither (off/off configuration, Figure $4 \mathrm{c}$ ), thus forming a wavelength selection device. The optical response of the ports of interest at the operation wavelengths is shown in Figure 5f. For comparison, the output intensities are normalized to the intensity of port A at wavelength of $\lambda_{0}=1520 \mathrm{~nm}$, which has the highest output intensity. The observed behavior is similar to that described in previous theoretical work where $2 \times 2$ and $3 \times 3$ junction arrays were designed to function as color routers based on the complex scattering coefficients of the junctions and the phase accumulated by wave propagation in isolated waveguides. ${ }^{2}$ There, it was found that the network parameters can be engineered to have a different set of resonances within the 
network for different wavelengths, causing light to route to different waveguide ports for different wavelengths.

The fabricated device is shown in Figure $4 a$, coupled from one of its port by the Si-ridge waveguide mode, which excites the CPP mode of the v-groove structure. The resulting nearfield intensity interference pattern for $\lambda_{0}=1520 \mathrm{~nm}$ is shown in Figure $4 b$, from which we can obverse that the network resonances result in selective coupling from the bottom input port $\left(P_{\text {in }}\right)$ into port A of the structure (on/off configuration). In Figure $4 \mathrm{~g}$ we plot the optical response of the ports of interest at $\lambda_{0}=1520 \mathrm{~nm}$, in good agreement with the on/off configurations obtained from the simulated device response (Figure 4f, black curve). We consider only the forward ports here as they exhibit sufficiently measurable intensity to allow for analysis without interference from the scattered light at the input interface.

It should be noted that a network with the full Boolean on/ off permutations in Port A and Port B (A:B on:off, off:on, on:on, and off:off) can be designed by modifying the dimensions of the overall resonator or the widths of the individual waveguides. To demonstrate such multiport operation, we simulated a resonant structure that is half of the size of the experimental structure in Figure 4 (with $7.5 \mu \mathrm{m}$ $\times 7.5 \mu \mathrm{m}$ dimensions). As seen in Figure 5, the intensity distribution of the structure with reduced resonator dimension for wavelengths of $1442 \mathrm{~nm}, 1480 \mathrm{~nm}, 1554 \mathrm{~nm}$, and $1610 \mathrm{~nm}$ are different in the two output ports of interest (ports A and B), showing that four on/off states can be achieved for different wavelengths with such an optimized RGWN structure. The $2 \times$ 2 RGWN can exhibit the full permutation of Boolean states in the two output ports of interest, unlike other photonic crystal/ plasmonic add/drop filters where only two output states (on/ off or off/on) can be realized. ${ }^{24,25}$ We note that it is even possible to employ all of the seven output ports in this $2 \times 2$ RGWN for complex signal routing by precise design of the resonant structure, leading to a more complex compact logic device (see Supporting Information Figure S5 for details). However, the full multiport operation of an RGWN is outside the scope of the present work and will be reported elsewhere.

Conclusion. In summary, we have experimentally demonstrated that resonant guided wave networks can be realized using plasmonic CPP v-groove waveguides, allowing for the design of ultracompact power splitters and logic devices. Selective coupling into the CPP v-groove waveguide mode is demonstrated, effectively filtering the SPP mode based on incident light polarization excited from silicon-on-insulator waveguides. This also constitutes a demonstration of a compact hybrid Si-photonic/v-groove plasmonic waveguide integrated circuit combining the low propagation loss advantage of $\mathrm{Si}$ photonics with the subwavelength modal confinement advantage of plasmonics.

Methods. Fabrication. The hybrid Si-photonic/v-groove plasmonic devices were fabricated on Si-on-insulator (SOI) wafers with a $220 \mathrm{~nm} \mathrm{Si}$ device layer on a $2 \mu \mathrm{m}$ buried oxide (BOX). Arrays of $100 \times 100 \mu \mathrm{m}^{2}$ Au pads were defined by electron-beam lithography using poly(methyl methacrylate) resist (MicroChem), where the resist layer was used both as a mask for $\mathrm{SF}_{6}$-based plasma etching of the top Si layer and, after partially etching the underlying BOX layer, as a liftoff layer for $\mathrm{Au}$ metallization. To define the metal depth for the v-groove waveguide, the chips were etched using buffered hydrofluoric acid solution to remove approximately $1 \mu \mathrm{m}$ of $\mathrm{SiO}_{2}$ from the BOX layer. A $900 \mathrm{~nm}$ Au layer was deposited into the etched regions by electron-beam evaporation. The SOI waveguides were patterned with a ridge width of $740 \mathrm{~nm}$ using negativetone electron-beam resist (Micro Resist Technology ma-N 2403), and the exposed $\mathrm{Si}$ was partially etched to a depth of 30 $\mathrm{nm}$ with a $\mathrm{C}_{4} \mathrm{~F}_{8} / \mathrm{O}_{2}$ plasma etching process. Diffraction grating couplers were etched to the same depth at the ends of the photonic waveguides to enable coupling from an off-chip source. Light from a tunable external-cavity diode laser (Santec TSL-210) was focused onto the grating couplers using a fiberpigtailed focuser, as described elsewhere. ${ }^{35}$ The v-groove channel waveguides were fabricated using multipass focused ion beam (FIB) milling on the Au pads at positions aligned with the SOI ridge waveguides. An interface FIB "cleaning” step was finally used to ensure maximum coupling from the SOI ridge waveguides to the $\mathrm{v}$-groove plasmonic waveguides, although this also added more separation between the waveguides. The resulting integrated structure consisted of a Si-ridge waveguide end-coupled to the v-groove Au waveguide with a waveguide separation of $\sim 200-300 \mathrm{~nm}$, depending on the device, and a vertical offset of $\sim-50 \mathrm{~nm}$ (Figure $1 \mathrm{c}-\mathrm{e}$ ).

Calculation of Coupling Efficiency. Placing the origin at some distance $L$ before the Si-slot waveguide meets the vgroove waveguide, the waveguide mode can be described by a sinusoid $A \mathrm{e}^{i\left(k_{1} z+\phi\right)}$, where $A$ is the real-valued amplitude of the wave, $k_{1}$ is the complex-valued Si-slot mode propagation wavevector, $z$ is the propagation direction toward the junction, and $\phi$ is some random phase. To this sinusoid, we add the phase it accumulates upon traveling to the Si-slot/v-groove waveguide junction $\mathrm{e}^{i\left(k_{1} L+\phi\right)}$, along with that of the reflection it encounters at that junction $r \mathrm{e}^{\mathrm{i} \phi_{r}}$, where $r$ is the real-valued reflection coefficient and $\phi_{r}$ is the phase picked up upon reflection, and the phase it accumulates at reaching the point $L$ from which the wave was originally measured, $\mathrm{e}^{-i\left[\mathrm{k}_{1}(z-L)\right]}$, where we have changed the sign of the sinusoid to indicate that it is propagating in the opposite direction as it was originally, and we have recentered the origin to the location of the Si-slot/vgroove waveguide junction. Putting it all together, we get a mode wave function of the Si-lot is described by the following expression:

$$
\begin{aligned}
& \Psi_{1}=\Psi_{\text {incident }}+\Psi_{L \text {-propagation }} \Psi_{\text {reflected }} \Psi_{L \text {-propagation,opposite direction }} \\
& \Psi_{1}(z)=A \mathrm{e}^{i\left(k_{1} z+\phi\right)}+A \mathrm{e}^{i\left(k_{1} L+\phi\right)} r \mathrm{e}^{i \phi_{r}} \mathrm{e}^{-i k_{1}(z-L)}
\end{aligned}
$$

Now, since we can only measure intensity, we take the amplitude squared of this expression to get the intensity at any point $\mathrm{z}$ along the Si-slot waveguide, before reaching the Si-slot/ v-groove waveguide junction, which equals the following after some simplification:

$$
I_{1}(z)=\Psi_{1} \Psi_{1}^{*}=A^{2}\left\{1+r^{2}+2 r \cos \left[2 k_{1}(L-z)+\phi_{r}\right]\right\}
$$

In this expression, $A^{2}$ gives a DC offset in the intensity profile, corresponding to the amplitude squared of the incident wave, which is what we are after, $r$ is related to the amplitude of the cosine function, which will come into play for later fits, and $L, k_{1}$, and $\phi_{r}$ are free fit parameters of the intensity function profile.

Thus, by fitting the intensity profile of the Si-waveguide before the waveguide, we get an intensity amplitude of $A^{2}=$ 0.5820 , which is related to how much power we sent via the $\mathrm{Si}$ slot waveguide mode into Si-slot/v-groove waveguide junction. Since there is a strict relation between amplitude squared of a mode and the power contained in the mode, i.e., $P \propto A^{2}$, which 
we find is equal to $P_{1}=0.0037 \times A^{2}$ from FDTD calculations, we measured the amount of power in the mode and related it to the intensity of the mode, which gave a proportionality constant of 0.0037 . Note that this is in general true for any point describing the Si-slot mode since we are describing a mode; thus we can take the intensity amplitude anywhere where there is a field and relate it to the amount of power contained within the mode.

Similarly, for the v-groove, since it is $30 \mu \mathrm{m}$ long, we can describe its mode wave function by a sinusoid $\Psi_{2}(z)=B \mathrm{e}^{i k_{2} z}$, where $B$ is the real-valued amplitude of the wave, $k_{2}$ is the complex-valued v-groove mode propagation wavevector, and $\mathrm{z}$ is the propagation direction away from the Si-slot/v-groove waveguide junction. Note that we have, in this case, omitted the random phase we had previously included for the Si-slot wave function since we are going to be taking the amplitude squared of this function. Thus, the intensity of the v-groove waveguide mode is given by the following expression:

$$
I_{2}(z)=\Psi_{2} \Psi_{2}^{*}=B^{2} \mathrm{e}^{-2 \operatorname{Re}\left\{k_{2}\right\} z}
$$

Again, by fitting the intensity profile of the v-groove after the Si-slot/v-groove waveguide junction, we get an intensity amplitude of $B^{2}=0.4784$, which is related to how much power is transmitted via the v-groove waveguide mode from the Si-slot/v-groove waveguide junction. In this case, we find from FDTD that $P_{2}=0.001 \times B^{2}$, a smaller power to intensity ratio that is not unexpected since the v-groove waveguide not only has smaller volume but also suffers from Ohmic losses.

Finally, with the extracted amplitudes of $A^{2}=0.5820$ and $B^{2}$ $=0.4784$ obtained from fitting the NSOM intensity profiles before and after the Si-slot/v-groove waveguide junction, which correspond to the amount of power incident onto and transmitted through the junction via the relations $P_{1}=$ $0.0037 \times A^{2}$ and $P_{2}=0.001 \times B^{2}$, we get that the amount of power coupled from the Si-slot waveguide into the v-groove waveguide is simply

$$
\eta=\frac{P_{2}}{P_{1}}=\frac{(0.001)(0.4784)}{(0.0037)(0.582)}=22.2 \%
$$

\section{ASSOCIATED CONTENT}

\section{S Supporting Information}

Experimental details on the fabrication and calculation details of the coupling efficiency. This material is available free of charge via the Internet at http://pubs.acs.org.

\section{AUTHOR INFORMATION}

\section{Corresponding Author}

*E-mail: haa@caltech.edu.

\section{Author Contributions}

S.P.B. and H.W.L. contributed equally.

\section{Notes}

The authors declare no competing financial interest.

\section{ACKNOWLEDGMENTS}

The authors thank Arian Kriesch and Ulf Peschel for inspiring discussions. This work was supported by the Multidisciplinary University Research Initiative Grant (Air Force Office of Scientific Research, FA9550-12-1-0024). H. W. Lee acknowledges by the Croucher Foundation of Hong Kong; S. P. Burgos acknowledges support from the National Science Foundation.
We acknowledge the use of facilities of the Kavli Nanoscience Institute (KNI) at Caltech.

\section{REFERENCES}

(1) Feigenbaum, E.; Atwater, H. A. Phys. Rev. Lett. 2010, 104, 147402.

(2) Feigenbaum, E.; Burgos, S. P.; Atwater, H. A. Opt. Express 2010, $18,25584-25595$.

(3) Feigenbaum, E.; Orenstein, M. Opt. Express 2007, 15, 1794817953.

(4) Yariv, A.; Yeh, P. Photonics: Optical Electronics in Modern Communications, 6th ed.; Oxford University Press: New York, 2006.

(5) Pavesi, L.; Lockwood, D. J. Topics in Applied Physics; Springer: New York, 2004.

(6) Dionne, J. A.; Lezec, H. J.; Atwater, H. A. Nano Lett. 2006, 6, $1928-1932$.

(7) Gramotnev, D. K.; bozhevolny, S. I. Nat. Photonics 2010, 4, 8391.

(8) Schuller, J. A.; Barnard, E. S.; Cai, W.; Jun, Y. C.; White, J. S.; Brongersma, M. L. Nat. Mater. 2010, 9, 193-204.

(9) Feng, N.; Brongersma, M. L.; Negro, L. D. IEEE J. Quantum Electron. 2007, 43, 479-485.

(10) Zia, R.; Schuller, J. A.; Chandran, A.; Brongersma, M. L. Mater. Today 2006, 9, 20-27.

(11) Cai, W.; Shin, W.; Fan, S.; Brongersma, M. L. Adv. Mater. (Weinheim, Ger.) 2010, 22, 5120-5124.

(12) Kriesch, A.; Burgos, S. P.; Ploss, D.; Pfeifer, H.; Atwater, H. A.; Peschel, U. Nano Lett. 2013, 13, 4539-4545.

(13) Feigenbaum, E.; Atwater, H. A. Opt. Express 2012, 20, 1067410683.

(14) Feigenbaum, E.; Burgos, S. P.; Atwater, H. A. In Photonic Crystals - Introduction, Applications and Theory; Massaro, A., Ed.; InTech: Hampshire, U.K., 2012.

(15) Lin, C.; Swillam, M. A.; Helmy, A. S. J. Opt. Soc. Am. B 2012, 29, 3157-3169.

(16) Feigenbaum, E.; Orenstein, M. J. Lightwave Technol. 2007, 25, 2547-2562.

(17) Moreno, E.; Garcia-Vidal, F. J.; Rodrigo, S. G.; Martin-Moreno, L.; Bozhevolnyi, S. I. Opt. Lett. 2006, 31, 3447-3449.

(18) Novikov, I. V.; Maradudin, A. A. Phys. Rev. B 2002, 66, 035403.

(19) Pile, D. F. P.; Gramotnev, D. K. Opt. Lett. 2004, 29, 1069-1071.

(20) Bozhevolnyi, S. I.; Volkov, V. S.; Devaux, E.; Laluet, J. Y.; Ebbesen, T. W. Nature 2006, 440, 508-511.

(21) Bozhevolnyi, S. I.; Volkov, V. S.; Devaux, E.; Ebbesen, T. W. Phys. Rev. Lett. 2005, 95, 046802.

(22) Volkov, V. S.; Bozhevolnyi, S. I.; Devaux, E.; Ebbesen, T. W. Appl. Phys. Lett. 2006, 89, 143108.

(23) Zenin, V. A.; Volkov, V. S.; Han, Z.; Bozhevolnyi, S. I.; Devaux, E.; Ebbesen, T. W. J. Opt. Soc. Am. B 2011, 28, 1596-1602.

(24) Qiang, Z.; Zhou, W.; Soref, R. A. Opt. Express 2007, 15, 18231831.

(25) Volkov, V. S.; Bozhevolnyi, S. I.; Devaux, E.; Laluet, J. Y.; Ebbesen, T. W. Nano Lett. 2007, 7, 880-884.

(26) Dionne, J. A.; Sweatlock, L. A.; Atwater, H. A.; Polman, A. Phys. Rev. B 2005, 72, 075405 .

(27) Veronis, G.; Fan, S. Opt. Express 2007, 15, 1211-1221.

(28) Kocabaso, S. E.; Veronis, G.; Miller, D. A. B.; Fan, S. Phys. Rev. B 2009, 79, 035120.

(29) Song, Y.; Wang, J.; Yan, M.; Qiu, M. J. Opt. 2011, 13, 075002. (30) Shi, P.; Zhou, G.; Chau, F. S. J. Opt. Soc. Am. B 2013, 30, 14261431.

(31) Zhu, S.; Liow, T. Y.; Lo, G. Q.; Kwong, D. L. Opt. Express 2011, $19,8888-8902$.

(32) Lau, B.; Swillam, M. A.; Helmy, A. S. Opt. Express 2010, 18, 27048-27059.

(33) Song, Y.; Wang, J.; Li, Q.; Yan, M.; Qiu, M. Opt. Express 2010, 18, 13173-13179. 
(34) Emboras, A.; Briggs, R. M.; Najar, A.; Nambiar, S.; Delacour, C.; Grosse, P.; Augendre, E.; Fedeli, J. M.; de Salvo, B.; Atwater, H. A.; Lamaestre, R. E. Appl. Phys. Lett. 2012, 101, 251117.

(35) Briggs, R. M.; Grandidier, J.; Burgos, S. P.; Feigenbaum, E.; Atwater, H. A. Nano Lett. 2010, 10, 4851-4857. 